\title{
Revealing the hidden lives of underground animals using magneto-inductive tracking
}

\author{
Andrew Markham and Niki Trigoni \\ Computing Laboratory \\ University of Oxford \\ firstname.lastname@comlab.ox.ac.uk
}

\author{
Stephen A. Ellwood and David W. Macdonald \\ Wildlife Conservation Research Unit \\ Department of Zoology, University of Oxford \\ firstname.lastname@zoo.ox.ac.uk
}

\begin{abstract}
Currently, there is no existing method for automatically tracking the location of burrowing animals when they are underground, consequently zoologists only have a partial view of their subterranean behaviour and habits. Conventional RF based methods of localization are unsuitable because electromagnetic waves are severely attenuated by soil and moisture. Here, we use an as yet unexploited method of localization, namely magneto-inductive (MI) localization. Magnetic fields are not affected by soil or water, and thus have virtually unattenuated ground penetration. In this paper, we present a method that allows the position of an animal to be determined through soil. Not only does this enable the study of behaviour, it also allows the structure of the tunnel to be automatically mapped as the animal moves through it. We describe the application for tracking wild European Badgers (Meles meles) within their burrows, providing experimental data from a two month deployment.
\end{abstract}

\section{Categories and Subject Descriptors}

H.4 [Information Systems Applications]: Miscellaneous

\section{General Terms \\ Design}

\section{Keywords}

Localization, Underground, Magneto-Inductive, Tracking

\section{Introduction}

There are a number of animals that burrow tunnels into the ground, primarily for shelter and protection while the animal is asleep. The size of the underground structure, however, varies considerably amongst species. Some animals, such as platypus (Ornithorhynchus anatinus), dig relatively simple burrows in stream banks, which are typically occupied by a single individual. Other animals, such as badgers

\footnotetext{
Permission to make digital or hard copies of all or part of this work for personal or classroom use is granted without fee provided that copies are not made or distributed for profit or commercial advantage and that copies bear this notice and the full citation on the first page. Copyrights for components of this work owned by others than ACM must be honored. Abstracting with credit is premitted. To copy otherwise, to republish, to post on servers or to redistribute to lists, requires prior specific permission and/or a fee.

SenSys'10, November 3-5, 2010, Zurich, Switzerland.

Copyright 2010 ACM 978-1-4503-0344-6/10/11 ...\$10.00
}

(Meles meles), excavate wide-ranging underground tunnel systems inhabited by a number of animals that form a social group [23]. Currently, there is no existing method for tracking the location of burrowing animals automatically when they are underground. In addition, tunnel structure can often only be determined by the extreme of excavation [23]. There is thus the need for a system which can localize animals while they are underground, in a non-invasive and automatic way.

In this paper we present a method, based on low frequency magnetic communication, which allows the position of a subterranean animal to be established. Not only does this enable the study of social interaction, it also allows the structure of the tunnel to be simultaneously mapped. We present a proof-of-concept application exemplified here by the tracking of European Badgers within their setts. The system overview is shown in Fig. 1.

Animals are equipped with collars that record the field strengths of coils of wire placed on the surface of the burrow. This data is compressed and stored until the animal emerges from its tunnel, at which point the buffered data is uploaded over a $2.4 \mathrm{GHz} 802.15 .4$ link. When underground, radio propagation from commodity nodes to surface devices is impossible due to severe attenuation, as tunnels can be as deep as $3 \mathrm{~m}$. The recorded magnetic field strengths are used to determine the animal's location using non-linear least squares estimation and knowledge of the spatial distribution of the magnetic field.

There has been a growing interest in recent years in underground communication networks [7, 8], but to the best of our knowledge localization has not yet been considered. In this work not only do we use MI based communication, we take it a step further and use MI based localization in an underground environment.

The key contributions of this paper are as follows:

- Hardware and firmware design of an MI based tracking system suitable for long-term deployment on wild animals.

- The first experimental demonstration of underground magneto-inductive localization of a sensor device.

- The first results showing how the fine-grained underground locations of wild badgers vary over time.

- Visualization of badger tunnel structure. 


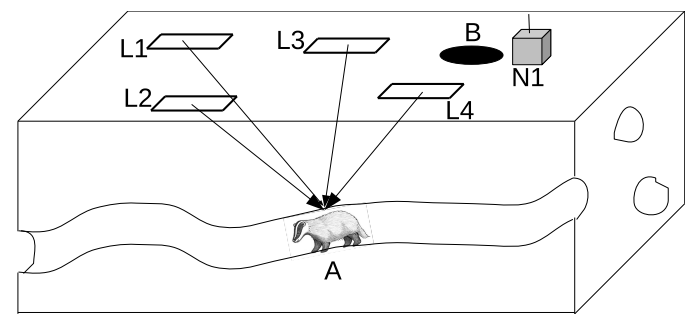

Figure 1. System overview showing an animal in its subterranean tunnel. The animal, at location $A$, receives and stores the magnetic field strengths associated with coils L1 through L4. As it is underground, radio communication is impossible. When it emerges at night from sett entrance $B$ to forage, the stored data is sent over an 802.15.4 radio link to a basestation, N1. Knowing the positions of the coils and the received field strength, locations of the animal over time can be determined.

\subsection{Paper structure}

First, we compare various localization modalities with particular reference to underground tracking in Sec. 2. The theory behind magneto-inductive localization is introduced in Sec. 3. followed by the design of our underground animal tracking system in Sec. 4. The principles behind MI localization are validated in Sec.5. In Sec. 6. we present results from our proof-of-concept deployment on four wild badgers. Similar work is highlighted and contrasted in Sec. 7, followed by a discussion of limitations in Sec. 8. Lastly, we summarize the core findings of our work in Sec. 9

\section{Comparison of various tracking approaches}

Localization is an important and necessary requirement in many sensor applications. In this section, we briefly overview different localization modalities, with particular reference to 'challenging' environments such as operation underground or underwater. We estimate the energy cost of the device being localized, using the product of the active time and current draw, under the assumption of a $3 \mathrm{~V}$ supply. The computational processing cost involved in determining the actual location is not considered, as for many devices the localization process is executed offline.

\subsection{GPS}

The Global Positioning System typically allows $5 \mathrm{~m} \mathrm{ac}-$ curacy to be obtained anywhere in the world. Its drawback (from a sensor perspective) is the energetical cost of acquiring a fix, especially if the device is started in cold or warm start mode. Typical power consumption of modern receivers running in the lowest power mode ( $1 \mathrm{~Hz}$ TricklePower) is still relatively high at $8 \mathrm{~mW}$, thus putting the energy per fix at approximately $3 \mathrm{mAs}$ (millamp seconds) [1]. Importantly, GPS cannot be used either underwater or underground and also is subject to large errors when operating under thick canopy due to multipath.

\subsection{RSSI localization}

This method which is popular in the WSN community involves a node recording the signal strengths of beacons and using this, along with a path-loss model, to estimate its location [21]. The advantage of this method is that it does not require an additional sensor, as the existing radio module is used. Furthermore, it allows for collaborative localization without the need for additional infrastructure. One of the problems with RSSI based localization is the effect of multipath and obstacles, which add a significant amount of noise to the signal strength measurements. As an example calculation, consider that a device listens to the medium for $50 \mathrm{~ms}$ in order to obtain beacon signals. With a typical radio receive current of $17 \mathrm{~mA}$, the energy consumption to obtain a fix is $0.85 \mathrm{mAs}$. Again, RSSI localization cannot effectively be used underground or underwater, because the radio signal is heavily attenuated. Other methods such as time-of-flight or angle of arrival localization suffer from the same problem of high signal attenuation.

\subsection{Acoustic localization}

This technique is based on the time-of-flight of an acoustic signal, typically in the ultrasonic portion of the spectrum [25]. Although this can be determined using two-way acoustic communication, a more common approach in the sensor network community is to simultaneously emit an RF and acoustic signal [25]. The one-way time-of-flight is proportional to the range between sensor devices, but also depends on ambient temperature which affects the speed of sound. This method has a much higher accuracy (in the order of $\mathrm{cm}$ ) than RF based localization, but has the drawbacks of requiring specialized hardware and having a limited range of a few metres due to attenuation of the acoustic wave. Assume that a device is able to obtain four ultrasonic range estimates in $100 \mathrm{~ms}$, and during this time both the ultrasonic and radio stages remain on, consuming $22 \mathrm{~mA}$ in total. Thus, the energy consumption per fix is approximately $2.2 \mathrm{mAs}$.

\subsection{Dead reckoning/IMU}

A method that allows devices to be tracked with a very high temporal resolution is inertial measurement or dead reckoning. The device is equipped with a triaxial accelerometer and magnetometer. By fusing these two types of information together, the displacement of the device can be calculated, relative to a starting position. The drawback of this method is that it suffers from squared error propagation due to the double integration of the accelerometer signal. This leads to cumulative error drift over time, and without a method for correction, will grow without bound. In addition, the sensor devices have to run continuously and generate a large amount of data that has to be retrieved somehow. Under the assumption of a $10 \mathrm{~Hz}$ position update rate, it takes 100 $\mathrm{ms}$ for a single fix. Accelerometers typically consume 300 $\mu \mathrm{A}$ and the magnetometer approximately $1 \mathrm{~mA}$. The energy per fix is thus $0.43 \mathrm{mAs}$. This method has been successfully used underwater [11].

\subsection{Magnetic Localization}

This technique is based on measuring the magnetic field strengths generated by low frequency loop antennas. The major advantage of this modality is that it is unaffected by the majority of materials found in natural habitats, such as soil and water. In addition, the field is non-propagating which has the implication that there are no multipath effects. This makes the signal strength measurements more accurate. However, the primary disadvantage is that it has a limited 
transmission range due to the fast field rolloff. The sensor technology itself is simple and the low cost. It consists of an ultra-low power receiver which measures the voltage induced in one or more pickup coils by the loop antenna. As an example, the AS3932 draws $8.3 \mu \mathrm{A}$ when actively detecting a transmission [2]. With a communication rate of 1300 symbols per second, obtaining magnetic field measurements from four coils takes under $0.4 \mathrm{~s}$. Thus the power per fix is $3.3 \mu \mathrm{As}$, which is two orders of magnitude smaller than dead reckoning. Magnetic localization can be used underground, underwater, and in air without requiring recalibration or channel modelling.

\subsection{Summary}

In summary, RF based methods suffer from an uncertain and highly attenuating communication channel making localization difficult or even impossible underwater or underground. Inertial tracking provides excellent short-term accuracy, but suffers from cumulative drift. Magneto-inductive localization, although inherently range limited, penetrates soil and water with negligible attenuation. It has the added benefit of ultra-low power consumption, making it highly useful in an animal tracking context. The following section discusses the theory behind MI localization.

\section{Magnetic Localization}

In this section, we first discuss factors affecting the spatial distribution of the magnetic field, such as coil geometry and size. This is then followed by an investigation into the effect of media such as water and soil on the magnetic field. Next we examine how to determine the location of a node, given a particular arrangement of coils.

\subsection{Spatial distribution of magnetic field}

The strength of the magnetic field at a particular location due to an arbitrary arrangement of current carrying conductors can be evaluated by determing the vector field potential and using the principle of superposition [12]. Alternatively the Biot-Savart law can be used if the geometry of the coil is simple to evaluate as a path integral (e.g. a circle). We present the first method here, as it can be generalized to any coil shape. Note that because the wavelength of the time varying field is very large $(\lambda=2400 \mathrm{~m})$ in comparison to the coil dimensions, the field can be regarded as being quasistatic, which simplifies the calculations. We provide a brief overview of the method - the details can be found in an elementary textbook on electromagnetics [12].

We consider the case of a rectangular current carrying loop in the $x-y$ plane, carrying a current of $I$, as shown in Fig. 2. We assume it has width $2 a$ and breadth $2 b$, and is centred at the origin. We first start by considering a small line segment $d l$ and evaluate its contribution to each component of the vector potential. Note that the z-component is zero and thus we only need to consider $A_{x}$ and $A_{y}$. Our aim is to calculate the magnetic field, $B(x, y, z)$ at a particular location in 3-D space, so that we can determine the voltages induced in a receiver device.

The $\mathrm{x}$-component of the vector potential can be expressed as:

$$
A_{x}=\frac{\mu_{0} \mu_{r} I}{4 \pi}\left[\int_{l=-a}^{l=a} \frac{d l}{R_{1}}+\int_{l=a}^{l=-a} \frac{d l}{R_{2}}\right]
$$

where

$R_{1}=\sqrt{(x-l)^{2}+(y+b)^{2}+z^{2}}$

$R_{2}=\sqrt{(x-l)^{2}+(y-b)^{2}+z^{2}}$

and $\mu_{r}$ is the relative permeability of the medium.

Similarly, the y-component is found to be:

$$
A_{y}=\frac{\mu_{0} \mu_{r} I}{4 \pi}\left[\int_{l=-b}^{l=b} \frac{d l}{R_{3}}+\int_{l=b}^{l=-b} \frac{d l}{R_{4}}\right]
$$

where

$R_{3}=\sqrt{(x+a)^{2}+(y-l)^{2}+z^{2}}$

$R_{4}=\sqrt{(x-a)^{2}+(y-l)^{2}+z^{2}}$

After evaluating the integrals in 1 and 2 the vector components of magnetic flux density are found by taking the curl of the vector potential.

$$
\vec{B}=\nabla \times \vec{A}
$$

In terms of the partial derivatives, this is expressed as:

$$
B_{x}=-\frac{\partial A_{y}}{\partial z}, B_{y}=\frac{\partial A_{x}}{\partial z}, B_{z}=\frac{\partial A_{y}}{\partial x}-\frac{\partial A_{x}}{\partial y}
$$

For reasons of space, the equations for each of the components of the magnetic flux are not stated here and can be found in [19].

We consider now the voltages induced at a receiver, situated at $[x, y, z]$, and equipped with three orthogonally mounted detection coils. The receiver is not necessarily oriented in the same way as the magnetic source, so we need to rotate the co-ordinate frame. This is undertaken using three rotational transformations, about the azimuthal, elevation and roll axes, denoted by $T_{\omega}, T_{\theta}$ and $T_{\phi}$ respectively. The magnetic field oriented to this reference frame is thus given by

$$
\vec{B}^{\prime}=T_{\phi} T_{\theta} T_{\omega} \vec{B}
$$

The magnitude of the voltage induced in each coil by the time varying field is given by Faraday's Law and is equal to

$$
\left|V_{x}\right|=\left|K \overrightarrow{B^{\prime}} \cdot \hat{i}\right|,\left|V_{y}\right|=\left|K \overrightarrow{B^{\prime}} \cdot \hat{j}\right|,\left|V_{z}\right|=\left|K \overrightarrow{B^{\prime}} \cdot \hat{k}\right|
$$

where $K$ is a multiplicative constant which accounts for the sensor gain, which is proportional to the number of turns and cross-sectional area of the coil. The combined magnitude of the three voltage components is given as

$$
\begin{aligned}
|V| & =\sqrt{V_{x}^{2}+V_{y}^{2}+V_{z}^{2}} \\
& =K\left|\overrightarrow{B^{\prime}}\right| \\
& =K|\vec{B}|
\end{aligned}
$$




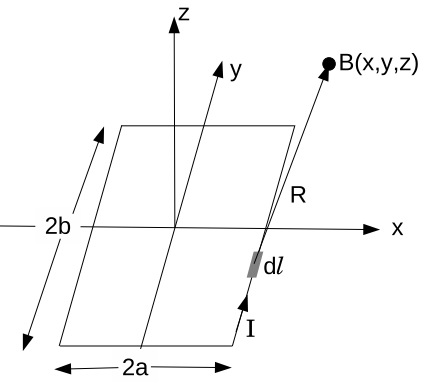

Figure 2. Geometry of a rectangular loop, centred in the $\mathrm{x}-\mathrm{y}$ plane.

Note that the rotation matrices do not affect the magnitude of the signal. Thus it can be seen that the magnitude of the voltage output from the triaxial sensor device is independent of its orientation and simply proportional to the magnitude of the field strength. Finally, this is converted to a decibel representation, using an implied reference voltage of $1 \mathrm{~V}$.

$$
\begin{array}{rrr}
d b_{\text {mag }} & = & 20 \log _{10}|V| \\
= & 20 \log _{10}|K \vec{B}| \\
= & G_{a}+20 \log _{10}|B|
\end{array}
$$

Note how the gain term emerges as an additive factor. This is the single factor that needs to be determined in order to calibrate each transmitter coil.

To account for coils which are not centred at the origin it is a simple matter to translate the coordinate frame before evaluating the field strength. For example, if the coil is displaced such that the centre is at $\left(x_{0}, y_{0}, z_{0}\right)$, then the following transformations should be made to Eq. 1 and 2 .

$$
x \leftarrow x-x_{0}, y \leftarrow y-y_{0}, z \leftarrow z-z_{0}
$$

If the coils are not mutually planar, a 3-D transformation matrix (consisting of a translational and rotational matrix) can be used to change the coordinate frame. For complex coil geometries, a similar potential field approach can be used to calculate the magnetic flux density, or finite element modelling can be used to determine the field patterns.

Although it is typical to consider forming an antenna from a loop of wire, it should be noted that a transmitting antenna can be made by injecting current directly into the earth, by driving in conducting stakes and applying a time varying current. This approach however has the drawback that the soil conductivity and moisture levels have an impact on the resultant magnetic field.

\subsection{Relative permeability}

The magnetic field, $B$, calculated in the previous section, is affected by materials to different degrees, depending on their relative permeabilities, denoted by $\mu_{r}$. A vacuum has a relative permeability of 1 . For ferromagnetic materials (such as iron or ferrite), the value of $\mu_{r}$ is very high (ranging from 80 to over 1000). However, non-magnetic materials, such as water, have a negligible effect on $\mu_{r}$, altering its value by only a few parts per million from unity. This means that soil (regardless of its volumetric water content) has no significant influence on an applied magnetic field. This has been confirmed experimentally in a number of studies, such as [20]. The negligible influence of water is a significant factor, because it also means that the magnetic field is not attenuated by the body of the animal.

The dielectric constant (relative permittivity, $\varepsilon_{r}$ ) is however very strongly affected by soil and water, in a complex, frequency dependent manner. This is why electromagnetic propagation through soil is particularly poor, as the electric field component (the E-field) of the wave is absorbed. This is something which has been noted by researchers experimenting with conventional nodes buried underground [26, 29].

\subsection{Localization techniques}

To solve the inverse problem, that of finding the location of the point in 3-D space given the field strengths, we use non-linear least squares estimation. We assume that the receiver is able to determine the magnitude of the magnetic field by using three orthogonally aligned receiving coils. From the measurements, we evaluate the residual as the difference between the predicted field and the measured field strengths (in dB). We use a standard Levenberg-Marquad gradient descent algorithm. Note that it is also possible to determine the relative orientation of the object by using a triaxial source of magnetic field [22]. This is useful if the pose needs to be established as well. However, as animal collars typically rotate around their necks we only consider position estimation.

Like other localization modalities, localization errors are a function not only of ranging errors, but also the geometry of coil layout. However, there is an important difference between RF based localization (which assumes a point source creating spherical or circular coverage) and MI based localization, in that the coil layout dictates the shape of the magnetic field in the near field. Altering the coil layout is a simple matter of changing the shape of the loop. Thus, irregularly shaped coils can be fabricated if necessary. What is interesting is that this can lead to simpler localization algorithms. For example, if the 2-D location of a sensor device was required, a grid of long, thin, antennas can be constructed arranged to form horizontal and vertical lines. Each antenna is driven in turn, and the location of the device is found at the intersection of the horizontal and vertical coils.

\section{System Design}

In this section, we present our MI based localization platform for tracking burrowing animals. We first specify the requirements of the system as proposed by the zoologists. We then introduce the components that make up the tracking system, discussing each of them in turn, with particular emphasis on steps that were taken to reduce the power consumption of the animal tag.

\subsection{System Requirements and Targets}

Based on consultations with the zoologists the following requirements for a generic underground animal tracking system were specified:

- The system must be able to determine the three dimensional location (to within $50 \mathrm{~cm}$ ) of a tagged animal. 


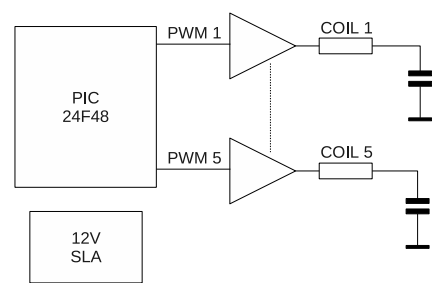

Figure 3. The antenna driver system.

- The system must be able to locate a tagged animal through at least $4 \mathrm{~m}$ of soil.

- Location estimates should have a temporal resolution of under a second.

- The devices attached to the animal must weigh less than $5 \%$ of the animal's bodyweight.

- The form factor of the tracking collar should prevent it from snagging underground.

- The devices attached to the animal should have an operational lifetime of at least three months.

- The devices attached to the animal must be relatively inexpensive.

- Based on the aggregated animal locations, the system must be able to construct a rough layout of the burrow structure of the animal.

\subsection{System components}

There are three main components in our system, namely the antenna drivers, the animal tags and the surface sensor network. As existing solutions were not available for low power MI localization, we had to custom design the tags and antenna system. In particular, the requirements specified in Sec. 4.1 imposed tight constraints on the allowable form factor and weight of the animal tag. Hence at each stage of the design process (prototype through to final PCB), input from the zoologists was sought in order that the completed module would be suitable for deployment on a wild animal. These led to some important design decisions, like choosing a wireless module for the tag that had an embedded antenna, rather than an external one. This is because burrowing animals can easily break an antenna, either by chewing it off or whilst burrowing. The functionality and design of each of these components will be discussed in turn.

\subsection{Antenna Driving System}

The antenna system generates the coded $130 \mathrm{kHz}$ magnetic fields which are used to localize the animals. Each driver unit is able to control up to five antennas or loops. A unit comprises a 12V 26 Ah battery, a PIC24F48GA002 microcontroller, half bridge TC4421 MOSFET drivers and the LC antenna units themselves. The antennas are formed from loops of wire which are tuned with removable capacitors to resonate with at the centre frequency. The system block diagram is shown in Fig. 3 .

The PIC microntroller generates Manchester coded PWM signals with a baud rate of 1300 symbols per second. Although the maximum transmission rate that can be detected by the animal tag is $4096 \mathrm{symbols} / \mathrm{sec}$ (i.e. $8192 \mathrm{bits} / \mathrm{sec}$ ),

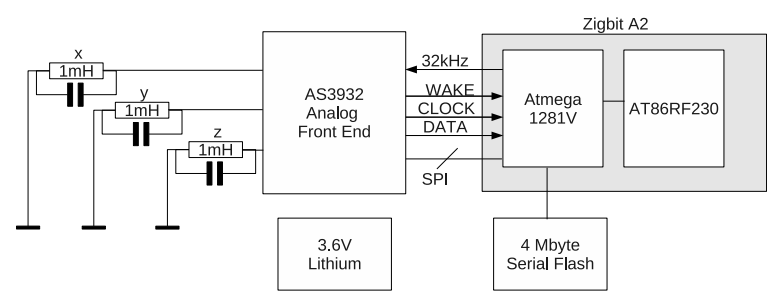

Figure 4. Components of the animal tag.

we operate at the default rate as it provides a good balance between noise immunity and transmission speed.

The transmitted message consists of the basestation and antenna ID ( 2 bytes), the timestamp ( 4 bytes) and a checksum (1 byte). This information allows the animal tag to determine which antenna transmitted the signal. In addition, the message is prepended with a correlation keyword, used to wake up the receiver. The maximum rate of transmission with these setting is $11 \mathrm{~Hz}$, which includes a small intersignal gap of $10 \mathrm{~ms}$. The signals for each antenna are timemultiplexed in a round-robin fashion so they do not interfere with one another. The design of the antenna driver unit is such that it can be easily controlled as part of the wireless network, with its parameters (such as number of active coils, scan rate and so forth) specified via the USART interface. Additionally, an antenna can be operated in full bridge mode, doubling the voltage swing, by connecting to it two half bridge drivers. The microcontroller takes care of the phasing requirements.

In order to compensate for small shifts in the resonant frequency of the LC tank circuit, the driving frequency is swept through a number of discrete frequencies in the range of the centre frequency. This allows the resonant antenna to 'lock' onto its own resonant frequency. The antenna driver circuitry consumes $1.2 \mathrm{~W}$ when operational.

\subsection{Animal Tag}

The system components of the animal tag are shown in Fig. 4. The core of the system is a Zigbit A-2 wireless sensor module, which consists of an Atmega- $1281 \mathrm{~V}$ microcontroller and an AT86RF230 2.4 GHz Zigbee compliant radio transceiver. The module has an onboard dual chip antenna which helps to simplify packaging. The signals from the three orthogonal coils are detected by the AS3932 low power programmable 3-D analog front end receiver [2], and buffered data is stored in a 4 Mbyte serial flash chip. The device is powered by an ER2450 lithium thionyl chloride battery. This battery was chosen primarily because it is packaged in a stainless steel case with a low magnetic signature and thus minimally distorts the magnetic field readings. We used the Contiki OS to run the code on the animal tags as it has off-the-shelf support for the Zigbit module.

\subsubsection{Analog Front End}

The analog front end (AFE) receiver is primarily used in the automotive industry, both in tyre pressure monitoring systems (TPMS) and passive keyless entry (PKE) units. The majority of TPMS analog front end chips are equipped with only a single input channel, but recently 3 -D receivers have been introduced which allow for the simultaneous detection 
of magnetic signals from three orthogonal axes. This is necessary in our application to find the magnitude of the magnetic field. This makes the magnetic field measurements rotationally invariant, as the orientation of the animal tag is an unknown factor. The orientation of the animal tag could be sensed through the addition of a triaxial accelerometer, but this would involve additional hardware and would increase the average power consumption. The AS3932 was chosen over other devices, such as the ATA5282, as it allows RSSI measurements from all three channels to be obtained automatically and simultaneously. In addition, it has a high sensitivity of $100 \mu \mathrm{V}$ compared to $2 \mathrm{mV}$ for the ATA5282. The drawback is that the quantization is fairly coarse at $+2 \mathrm{~dB}$ per step, with each channel only having a 5 bit resolution. Thus, the dynamic range of the AFE is only $64 \mathrm{~dB}$.

Each channel consists of a small $1 \mathrm{mH}$ transponder for use in TPMS and a $1.5 \mathrm{nF}$ resonant capacitor. The resonant frequency of the LC tank circuit is $130 \mathrm{kHz}$. The transponder has a ferrite core, resulting in a Q-factor of 27, which helps to increase sensitivity, given the small cross-sectional area of the transponder. Initially, we used X7R chip capacitors, but we found that their temperature stability was poor. We thus switched to slightly more expensive COG (constant-overgrade) capacitors which have a virtually zero temperature coefficient. An added advantage is that the dielectric losses are lower which resulted in an additional $+2 \mathrm{~dB}$ of sensitivity. We attempted to source flat, integrated triaxial transponder coils, but were unable to purchase them in small quantities. Instead we used three identical transponder coils. The drawback of this is that the $\mathrm{z}$-axis coil is mounted perpendicular to the circuit board, which increases the height of the device. To reduce component count, we use the $32.768 \mathrm{kHz}$ oscillator output from the Zigbit module to provide an accurate clock for the AFE. Thus, the entire MI sensor consists of one AFE chip, three capacitors and three inductors. This helps to keep the cost of the system down.

The active current consumption of the AFE is extremely low at $8.3 \mu \mathrm{A}$. The standby current (waiting for the correlation keyword) is $2.7 \mu \mathrm{A}$. The AFE generates a pin change interrupt when a valid correlation keyword is detected, waking up the Zigbit microcontroller. The AFE can be configured to either output the raw bitstream or the Manchester decoded stream. Although Manchester encoding halves the symbol rate, it allows for the recovery of the clock signal. Thus the microcontroller does not need to use an asynchronous USART which requires clocking. The data is sampled on the rising edge of the clock signal which is generated by the AFE. Again, this is done by using a pin change interrupt.

Once a tag has been read and error-checked, the stored RSSI values are retrieved from the AFE and converted to the magnitude. The magnitude (in $\mathrm{dB}$ ) is further scaled by a factor of 4.0 to use the widest dynamic range possible and converted to an unsigned 8 bit integer. Although this results in a slight loss of precision, it helps greatly to reduce the memory usage and allows us to use an efficient compression algorithm.

To further reduce the power consumption, we modified the Contiki kernel. Typically, when an interrupt occurs (either from the timer or another source), the ISR is serviced and then all outstanding events are processed. However, in our application, an interrupt is triggered on every falling edge of the clocked data from the AFE, which leads to a large amount of unnecessary servicing of process threads. We thus use a flag to only take the CPU out of sleep mode when either a complete tag has been read or any interrupt other than the AFE serial clock occurs. This helps to significantly reduce the current consumption from $300 \mu \mathrm{A}$ when detecting a signal from an antenna to $110 \mu \mathrm{A}$.

\subsubsection{Wavelet Compression}

An animal tag, when in range of multiple antennas, generates a large amount of data. The worst case scenario is if it detects all 11 transmissions per second. Each packet consists of 6 bytes of data (the antenna's ID and the timestamp). In addition, the record is also appended with magnitude of the RSSI. If this data was stored natively, this would require 77 bytes a second. This equates to over 3 Mbytes per 12 hour day. Thus if the animal does not emerge that night, this will lead to data loss as the memory is only 4 Mbyte.

There is however a lot of similarity between successive records from the same antenna. The antenna ID will remain constant, so only needs to be stored once in a base record. The timestamps will be monotonically increasing, thus the initial timestamp can be also stored in the base record. The magnitude of the magnetic field is stored in a buffer of length $\mathrm{N}$, where each entry corresponds to a successive time interval. This simple means of grouping data provides a compression factor of approximately seven-fold, reducing the data load to approximately 475 kbytes per day. Although this is a great improvement, this only allows for approximately one week of data to be stored on a collar. Whilst animals typically emerge every day, in winter there are periods where they are torpid and can remain underground for a few days at a time. This will again result in a potential data loss.

In order to further minimize the amount of data that needs to be sent over the wireless link, and also limit the energy consumed in storing data within the flash, we used an inplace Haar wavelet transformation [10] and Elias-Gamma encoding. The integer lifting Haar wavelet transform decomposes data into spectral (or detail) components, and is much more computationally efficient than the FFT. The additional advantage of the lifting transform is that it allows for in-place computation which means that no additional buffer space is required for compression, as the original array is overwritten with the compressed data. The wavelet transform on its own does not compress data. It does however cluster data into detail bands, roughly corresponding to frequency bands. In tests, it was found that the majority of the highest level detail coefficients were zero, so these were removed, giving an initial compression factor of two. We then used Elias-Gamma encoding, which is an integer based prefix code, to compress the Haar coefficients. Thus, each coefficient is stored using only a few bits of information, with a coefficient of zero being stored using only one bit.

Typically, the overall compression factor that is obtained in tests, compared with storing the raw data ( 7 bytes per entry) is approximately 23 . This reduces the worst case amount of data to approximately 130 kbytes per day. This allows almost a month of data to be stored in the flash memory, 


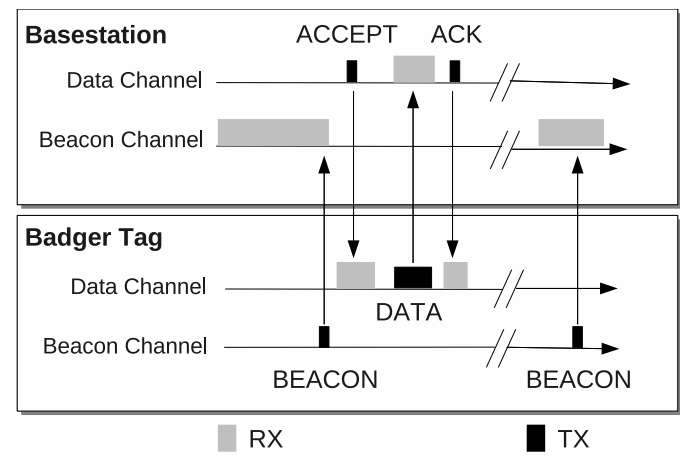

Figure 5. The simple, multichannel beacon protocol showing how beacons are used to initiate data transfers.

eliminating the potential for data loss. In addition, the compression helps greatly to reduce energy consumption both in saving the data to the flash in the first place and in sending the buffered data over the radio link.

\subsubsection{Beacon protocol}

The purpose of the beacon protocol is to allow animal tags to rapidly download their buffered data when they emerge at night. As an animal tag can generate over 100 kbytes a day, it is imperative that the data be collected quickly and reliably. An overview of the beacon protocol is shown in Fig. 5 showing how beacons are used to schedule data transfers. In our system, beacons are transmitted every $10 \mathrm{~s}$. The tag listens for ACCEPT messages for $15 \mathrm{~ms}$ before returning to sleep mode. If an ACCEPT message is received, data is transferred to the basestation. To allow for multiple basestations, ACCEPT messages are transmitted with a random dither to prevent collisions at the receiver. The animal tag will respond to the first message received.

The major advantage of this protocol is that data is transferred rapidly and without the overhead of a complex MAC protocol. Interference between nodes is eliminated by the simple step of using a dedicated channel just for transmitting beacons and randomly chosen channels which are used for data transactions. Thus a bulk download by a tag, which could take $30 \mathrm{~s}$ to complete, does not lock out the medium or get subjected to interference by other animal tags. If the data channel is busy or being used by another device, the data forwarding will fail. However, when it next broadcasts a beacon, the animal tag will choose a random data channel, so the probability of persistent interference is small.

Data is transferred in 64 byte blocks, with 16 blocks comprising a 512 byte page in the dataflash. Each block is acknowledged to ensure reliable delivery. This is especially important because the compressed sensing records are not necessarily aligned with block boundaries. Block loss will lead to data which is impossible to decompress. Each block is allowed to be retransmitted three times before the process is aborted. In the case of an acknowledgement failure, the block index is rolled back to the start of the current page. Once all the data has been uploaded, the animal tag only sends beacons and does not switch to the data channel. This continues until it has a minimum threshold of data available to upload (1.5 kbytes in our case). This helps to further save energy when the animal has no data to send.

Because of the simplified protocol with a focus on efficient transfer of information, the animal tags are able to transfer data reliably at a rate of $3.5 \mathrm{kbytes} / \mathrm{sec}$, which includes the overhead of reading and writing to flash. With a selective ARQ protocol, this rate could be further increased if data was only acknowledged at the page level, not at the individual block level. Note that the beacons serve a dual purpose of data transfer initiation and also allow for the detection of the position of animals when they are above ground, either using RSSI or simple proximity measurements.

\subsubsection{Power budget}

For an application like this, where devices are deployed on wild animals, it is desirable that tags run for as long as possible. As an example, badger trapping can only occur from June to December to avoid disturbing breeding animals or animals with dependent young. Trapping is also very labour intensive as it requires 8 people to work from dawn (to collect badgers from the traps and sedate them) to dusk (to bait traps with peanuts for the night and release the badgers) for about 10 days [17]. Thus, there has been a strong focus on optimizing the power consumption of the animal tag, shifting power usage to surface based components which can be easily maintained.

The breakdown of energy consumption amongst the various modules in the animal tag is shown in Table. 11. This is further split into energy consumed during the day whilst the animal is underground, and energy used at night when the animal is foraging. The table demonstrates that the majority of the energy is consumed by the microcontroller itself whilst in comparison the energy used by the AFE is negligible. The beacons sent every $10 \mathrm{~s}$ also consume a relatively large proportion of energy. Using these figures, and the specified $1000 \mathrm{mAh}$ capacity of the battery, the expected tag lifetime is approximately 150 days or 5 months, which satisfies the requirements of the zoologists. The table demonstrates that the microcontroller itself is the main cause of energy consumption. In our case, this is because we have to maintain an asynchronous clock for the pin change interrupt from the AFE, which prevents us from placing the microcontroller into deep sleep mode, which would only use $6 \mu \mathrm{A}$ when idle, as opposed to $60 \mu \mathrm{A}$. This suggests that there is significant room for improvement in tag lifetime by using a more power efficient microcontroller 1

\subsection{Surface components}

The basestation is placed close to the sett and is powered by the same battery as the antenna driver. This allows it to listen for incoming beacons continuously. When a beacon is heard, it switches to the assigned data channel and initiates the transaction, saving the data in SD card based flash. All data is time-stamped and the LQI and RSSI are

\footnotetext{
${ }^{1}$ We have implemented an ultra-low power datalogger version of the tag (no radio) which uses a PIC24F48GA002 microcontroller. By clocking the microcontroller at $32 \mathrm{kHz}$, we are able to drop the average current consumption for the entire module to $20 \mu \mathrm{A}$ when detecting tags. Using the same battery, this would result in a 6 year lifetime, which exceeds a typical badger lifetime of 3-5 years.
} 
Table 1. Breakdown of energy amongst the various components.

\begin{tabular}{|l|l|l|r|l|l|r|}
\hline \multirow{2}{*}{ Module } & \multicolumn{3}{|c|}{ Day (underground) } & \multicolumn{3}{c|}{ Night (aboveground) } \\
\cline { 2 - 7 } & Current & Time & Energy $(\mathrm{mAh})$ & Current & Time & Energy (mAh) \\
\hline AFE & $10 \mu \mathrm{A}$ & 12 hours & 0.12 & $2.7 \mu \mathrm{A}$ & 12 hours & 0.03 \\
\hline Dataflash & $30 \mathrm{~mA}$ & $30 \mathrm{~s}$ & 0.25 & $30 \mathrm{~mA}$ & $30 \mathrm{~s}$ & 0.25 \\
\hline Beaconing & $17 \mathrm{~mA}$ & $130 \mathrm{~s}$ & 0.82 & $17 \mathrm{~mA}$ & $130 \mathrm{~s}$ & 0.82 \\
\hline Data transfer & 0 & 0 & 0 & $17 \mathrm{~mA}$ & $30 \mathrm{~s}$ & 0.14 \\
\hline Microcontroller & $200 \mu \mathrm{A}$ & 12 hours & 2.4 & $150 \mu \mathrm{A}$ & 12 hours & 1.8 \\
\hline Total & \multicolumn{5}{|c|}{} \\
\hline Daily Total & \multicolumn{7}{|c|}{} & 3.59 & & 3.04 \\
\hline
\end{tabular}

also recorded. The basestation is also equipped with a temperature sensor which measures the ambient air temperature.

In order to demonstrate that the animal tags could be integrated into a conventional wireless sensor network, we also implemented surface tracking nodes which listen for incoming beacons and forward them to the basestation. The surface tracking nodes are standard Tmote SKY nodes. These nodes listen for incoming beacons and forward them to the basestation. We used the RIME tree collect module of Contiki and $\mathrm{X}$-MAC to forward the data to the basestation. We use a separate channel from the set used by the animal tags in order to prevent any interference. In order to conserve power, the radio transceivers were powered off during the day. Although we could have used a clock signal from the basestation, we used a much simpler approach which monitored the ambient light level and turned off the radio when it exceeded a threshold (i.e. the radio was off during daylight). The radio was duty-cycled such that it listens for beacons for three seconds every ten seconds. When a node detects an animal tag, it then transmits dummy beacons to wake up its neighbouring nodes, and then remains active for as long as the tag is present in its radio range. When a node is not listening for beacons, it switches to X-MAC, waiting to forward data.

\section{Calibration and Validation}

In this section, we confirm that the basic concepts of MI localization are correct and that the technology does indeed work through soil. We performed tests in an underground pipe, as inserting a device within an inhabited badger sett could disturb denning behaviour, potentially causing a badger to disperse from its home sett.

\subsection{Validation of predicted field}

In Sec. 3, the equations which specify the spatial distribution of the magnetic field around a coil were presented. We show here that the measured field agrees with the predicted field. For this experiment, we used a single turn coil of dimensions $5 \mathrm{~m}$ by $1 \mathrm{~m}$, which was buried to a depth of $5 \mathrm{~cm}$ underground. This is identical to the final setup in Sec.6 6and we used an assembled animal tag with triaxial pickup coils, as described in Sec. 4 to measure the magnitude of the magnetic field. The calibration process consists of taking three measurements of the magnetic field (e.g. at $1 \mathrm{~m}, 2 \mathrm{~m}$ and $3 \mathrm{~m}$ from the coil) and using linear regression to determine the additive gain factor, $G_{a}$ in Eq. 7. by comparing the measured and predicted values of the field strength at the known locations.

Once the gain was determined for this particular coil, the

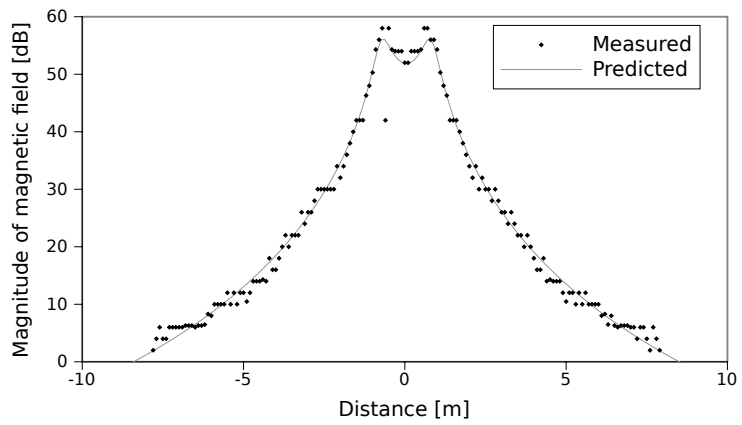

Figure 6. Comparison between measured (dots) and predicted (solid line) fields for a large coil.

magnetic field was measured at $10 \mathrm{~cm}$ intervals along the coil, at a height of $1 \mathrm{~m}$. The results are shown in Fig. 6 showing that there is a very good correspondence between the measured and predicted fields. The standard deviation of the error was found to be $0.98 \mathrm{~dB}$. The two peaks in the graph occur when the badger tag is directly above the current carrying wire of the antenna. Note however that the measurements for strong field strengths underestimate the real field - this is possibly due to saturation of the ferrite cored transponder antennas, as a result of the nonlinearity inherent in the core magnetization.

\subsection{Underground test}

In order to confirm that MI localization works in an underground setting, we placed five transmitting coils above a buried concrete drain pipe with diameter $300 \mathrm{~mm}$. Each coil was $20 \mathrm{~cm} \times 30 \mathrm{~cm}$ in size, and had a horizontal range of approximately $4 \mathrm{~m}$. Each coil was calibrated using the method in Sec. 5.1. The drain pipe was buried at a depth of $1.1 \mathrm{~m}$ and was approximately $5 \mathrm{~m}$ long. A badger tag was dragged through on a line and 100 magnetic field readings were taken at $1 \mathrm{~m}$ intervals. It should be noted that the pipe was half full of mud and debris. When the tag was fully inside the pipe radio communication with the surface was impossible, except if the receiver was also placed within the pipe. This confirms that there was significant attenuation of the radio signal due to soil, as expected.

The results from the trial are shown in Fig. 7. Note that although 100 readings were taken at each location, the estimated locations do not form a dense scatter plot, but rather are concentrated at discrete points. This is due to the coarse quantization of the analog front end used in the animal tag. This could be overcome by randomly dithering the output 


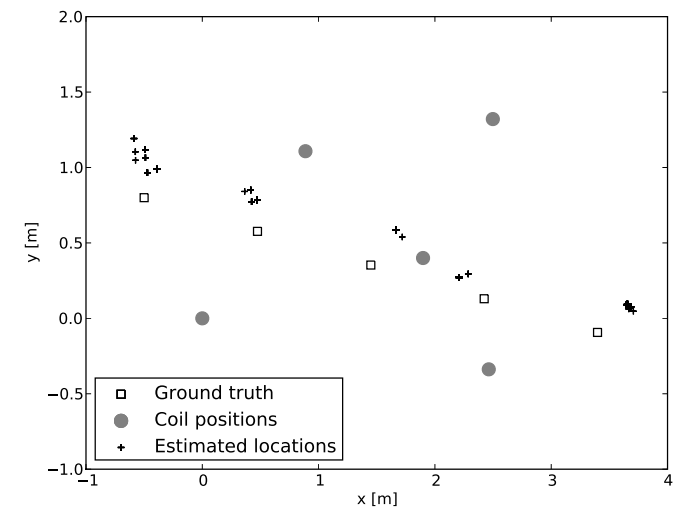

Figure 7. Results from the underground pipe test.

power of each transmitter coil, which can easily be accomplished by altering the pulse-width.

The magnitude of the ranging error in the horizontal plane is $0.26 \pm 0.10 \mathrm{~m}$ and in the vertical plane is $0.34 \pm 0.30 \mathrm{~m}$. The CDF of absolute localization errors is shown in Fig. 8 The accuracy is poorer in the vertical plane because the coils are co-linear and aligned in the same plane. This test demonstrates that accurate 3-D localization through the earth is possible using magnetic fields.

\section{Real world application: badger tracking}

This section discusses our real world deployment on four wild adult badgers. First, we discuss the current approaches to determining badger behaviour. Next, we overview the process involved in fabricating the collars and attaching them to the badgers, followed by a summary of the overall performance of the system over a two month period, briefly outlining some issues that were faced during the deployment. Finally, we present some badger traces from the deployed system.

\subsection{Are badgers "sett" in their ways?}

Badgers are fossorial carnivores that typically live in woodlands. Their behaviour can be monitored above ground using established techniques, but due to the difficulty in monitoring them underground comparatively little is known about their subterranean behaviour. As they spend more than half their lives underground, this results in incomplete knowledge.

Badgers are the subject of considerable controversy with regards to their possible role in the spread of bovine tuberculosis [18]. Better understanding of badger behaviour and activity is thus essential to make informed management decisions.

Badgers live in communal burrow systems (often referred to as setts). These setts typically consist of multiple entrances (typically around 5-15) leading to a connected underground series of tunnels [23]. These tunnels terminate in large chambers, where badgers rest during the day. Tunnel diameter is generally $25-30 \mathrm{~cm}$ (i.e. one adult badger in width) and depth is up to $4 \mathrm{~m} \mathrm{[23].}$

The existing approach to monitoring badger and other fossorial animal activity underground is very labour intensive. The typical method is to equip the badgers with VHF radio

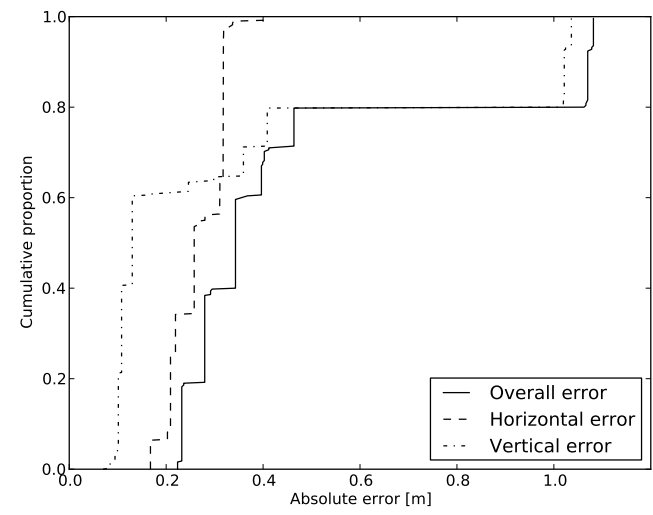

Figure 8. Cumulative distribution of localization errors.

collars which emit high power beacons every few seconds [24]. Researchers use high gain Yagi antennas connected to a sensitive radio receiver and estimate their location underground by triangulation. This process is repeated for all the collars in the study, at all the possible setts under investigation. This method results in coarse location estimates (typically in the order of $1 \mathrm{~m}$ ). In addition, there are areas which cannot be easily surveyed e.g. if there is thick vegetation. The larger problem with this technique is however that typically only one fix per animal per day can be obtained. Thus, the temporal resolution is poor. Lastly, walking over the top of the sett, while making a fix is likely to disturb the animal, possibly causing it to move or alter its behaviour.

Of further interest to zoologists is the sett architecture and geometry as it is related to the issue of co-operation and social structure within a sett. There are a number of methods to determine this, varying from the extreme of excavation (which destroys a sett) [23] to techniques more commonly used in archaeology. These include ground penetrating radar (GPR) and electrical resistance tomography (ERT) [9]. All these techniques involve disturbance to the sett and cannot actually be used to track animal location.

Based on this outline, it can be seen that there is a strong case for providing a continuous, non-invasive means of determining badger location and activity underground. Such a tool would greatly aid zoologists in obtaining a detailed record of subterranean badger behaviour. In addition, the general area of mapping tunnel structure is also an area which warrants investigation. It is these key issues that we seek to address.

\subsection{Collar fabrication and attachment}

An assembled badger tag and its battery are shown in Fig. 9 (a). The dimensions of the tag are $39 \mathrm{~mm}(\mathrm{l}) \times$ $22 \mathrm{~mm}(\mathrm{w}) \times 12 \mathrm{~mm}(\mathrm{~h})$. The figure shows three orthogonally mounted transponder coils and the Zigbit module. The dataflash and AFE are mounted on the underside of the PCB. In order to prevent eddy currents that would affect the magnetic field readings, the ground plane underneath the transponder coils has a gap in it.

The tag was mounted on a leather collar and potted using epoxy resin. This was milled to remove the excess resin and sharp edges, making it suitable for attaching to a badger. A 


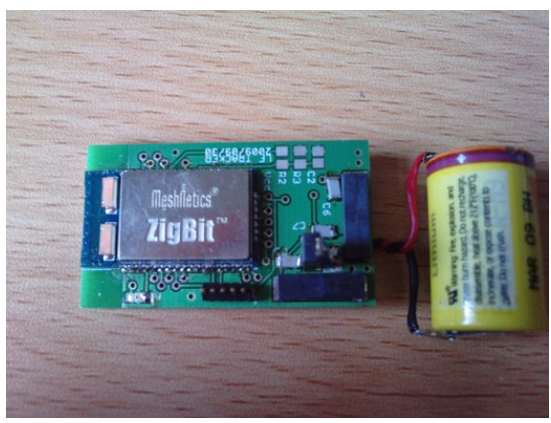

(a)

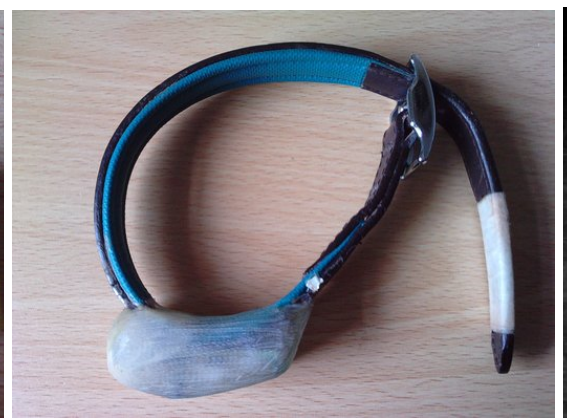

(b)

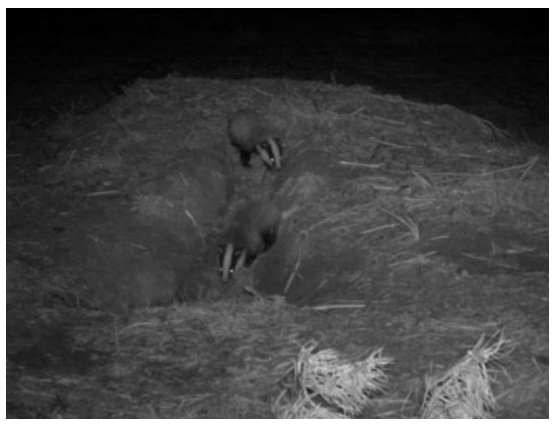

(c)

Figure 9. Stages involved in the badger tagging process. (a) An assembled badger tracking board. (b) A tracking collar with the tracking board encased in epoxy resin. (c) Night-time footage of two badgers.

completed collar is shown in Fig. 9 (b). The average total weight of an assembled collar is $105 \mathrm{~g}$, which is less than $2 \%$ of the weight of an $8 \mathrm{~kg}$ average sized badger. Note that the unit is self-contained and there are no protruding objects such as an external antenna. The overall cost of an assembled tracking collar is approximately $\mathrm{U} \$ 80$, which satisfies the low cost requirements.

Trapping and collaring was undertaken in January 2010 by experienced zoologists operating under Home Office Licence 30/2138 and Natural England Licence 20001537 in Wytham Woods, Oxfordshire. Although more than four collars were available, the sub-zero temperatures at the time of trapping reduced badger activity and thus only five badgers in total were caught at the sett of interest. One of the badgers was a cub from the previous spring and could not be collared. Of the remaining badgers, two were adult males and two were adult females, and these were fitted with collars under sedation. After collaring, badgers were kept in a warm recovery room before being released at the same location where trapped [17].

\subsection{System calibration}

Initially, we deployed five antenna units consisting of 35 turns of $0.5 \mathrm{~mm}$ diameter copper wire wrapped around a 20 $\mathrm{cm} \times 30 \mathrm{~cm}$ plastic former. They were connected to the antenna driving unit using twisted pair wire which prevents leakage of the magnetic flux from the connecting cable itself. This resulted in an antenna with a very high Q-factor, approximately 100 . Although these worked well in early tests, we found that in the field there were significant variations in field strength over time. This is due to variations in the series tuning capacitor with temperature. Due to the high-Q factor (and correspondingly narrow operating bandwidth), even a $5 \%$ variation in capacitance leads to a $10 \mathrm{~dB}$ reduction in the current flowing through the antenna. This prevented us from accurately being able to estimate the badger's position. However, it did enable us to determine if a badger was in proximity of an antenna.

In order to solve this problem, we switched to using antennas with a lower Q-factor which have a better tolerance. The existing high-Q antennas were replaced with four very large, single turn coils made from $1.0 \mathrm{~mm}$ diameter single core insulated cable, typically used in mains wiring.

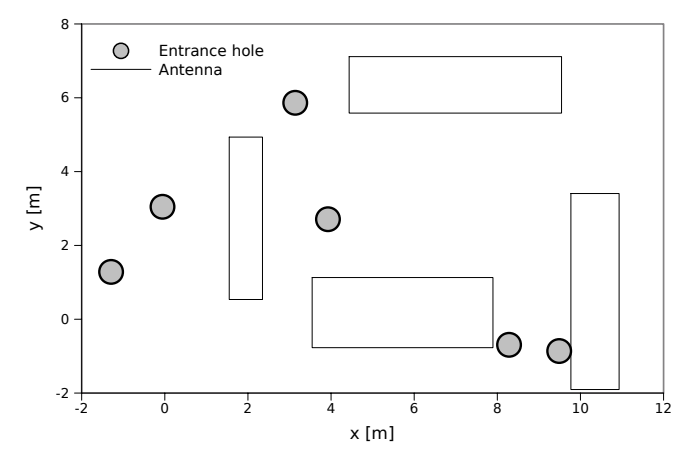

Figure 10. Layout of the large loop transmitting antennas at the badger sett. Also shown are sett entrance/exit holes.

Each antenna forms a rectangular loop approximately $5 \mathrm{~m}$ in length and $1 \mathrm{~m}$ in width. The Q-factor of these larger antennas was approximately 10 . Note that a $5 \%$ variation in tuning capacitance only leads to a $0.5 \mathrm{~dB}$ reduction in antenna current, as the bandwidth of the resonant circuit is much wider. This makes long-term antenna operation much more reliable, and also makes antenna tuning simpler as the choice of tuning capacitor is no longer critical. These antennas are simple to deploy, as they are just buried within a slit trench, at a depth of approximately $5 \mathrm{~cm}$. The advantage of this setup is that it is more robust to disturbance by the badgers. Over the course of the deployment, we found that portions of the loop were occasionally dug up by the badgers looking for earthworms but the badgers did not break the heavy gauge cable. No other animals appeared to disturb the antennas.

By increasing the area of the coil we were able to cover a larger area, with each loop having a horizontal range of approximately $9 \mathrm{~m}$. We also placed a large antenna around the periphery of the sett which serves as a simple presence/absence detector. This coil has an approximate radius of $12 \mathrm{~m}$ and was tethered to convenient tree branches and trunks, approximately $2 \mathrm{~m}$ above the ground, so as to not pose a risk to other animals such as deer. It should be noted that the first phase deployment of the small antennas was actually very useful, as it allowed us to determine the locations where badgers were denning. Thus when we came to install 
Table 2. Overall performance of the deployed badger tags

\begin{tabular}{|l|l|r|r|r|r|}
\hline Tag ID & Badger Sex & Collar Runtime (days) & Number of readings & Number of bytes & Compression Factor \\
\hline A & F & 34 & 290777 & 84544 & 24.1 \\
\hline B & M & 68 & 4354978 & 1260736 & 24.2 \\
\hline C & M & 42 & 1595042 & 456896 & 24.4 \\
\hline D & F & 42 & 774933 & 269824 & 20.1 \\
\hline
\end{tabular}

the large, buried antennas, we were able to place them to cover the most utilized areas.

In order to obtain the badger traces, we first had to calibrate our system. This involved surveying all the transmitting coils and badger holes using a laser range finder. These ranges were then converted to distances using nonlinear least squares fitting. Next, we took readings of field strengths at known locations and used these to determine the gain factor $G_{a}$ for each antenna. The locations of the large antennas and the positions of badger holes are shown in Fig. 10

Although the larger coils allowed us to cover a greater area, the result was that the vertical accuracy of the system was negatively affected. Whereas in our initial system the vertical (depth) accuracy was in the order of $30 \mathrm{~cm}$, in this system, the accuracy is in the region of $80 \mathrm{~cm}$. However, the horizontal accuracy remains similar, with an average error of $35 \mathrm{~cm}$. Thus, although we can estimate the positions of the badgers, we are unable to accurately determine their depth, as was possible in the tunnel test in Sec.5.2. Again, it must be stated that the accuracy obtained is still considerably better than the approximate $1 \mathrm{~m}$ currently achievable using VHF tracking.

\subsection{Overall system performance}

A summary of the performance of the system is shown in Table 2. The total number of bytes sent by each collar is also shown in the table, along with the number of magnetic field readings. The compression factor (relative to the raw data) achieved by the real world system is similar to what was predicted in Sec. 4.4.2. The simple beacon protocol transferred data reliably at an average throughput of $4.1 \mathrm{kbytes} / \mathrm{sec}$, without any dataloss (i.e. all generated data was successfully downloaded).

The antenna driving system (and basestation) worked consistently throughout the study period, but there were two interruptions, each lasting a few days, when the equipment was interfered with by the badgers. The solution to this was to suspend the battery, antenna driver and basestation from a tree such that they were out of reach.

As can be seen none of the collars achieved the design lifetime of three months of operation. However, an identical collar which was left running indoors was still functioning at the time of writing (91 days), with a stable battery voltage of $3.50 \mathrm{~V}$. The reasons for collars not achieving the required lifetime is not clear, as collars can be destroyed or removed by the badgers, in addition to a hardware or software failure. However, based on the battery voltage transmitted in the beacon message, it appears that at least two of the collars failed as a result of passivation of the lithium cell. Passivation is a particular issue with lithium thionyl chloride cells, as opposed to the more commonly used lithium man- ganese and refers to the buildup of crystals around the anode, leading to a voltage delay i.e. when the node draws a large amount of current, the terminal voltage collapses for a few seconds. Over a period of time (hours or days), the battery voltage slowly recovers, but there is a risk of the processor being stuck in a brownout loop which rapidly exhausts the battery. This effect is exacerbated by low temperatures, and failures did occur when ambient temperature was $<0^{\circ} \mathrm{C}$. It is possible to depassivate a battery by periodically drawing a large current, which removes the buildup of insulating crystals around the anode.

\subsection{Individual Badger Traces}

In Fig. 11 trajectories of individual badgers, grouped into hourly sets of locations are shown. These capture how the badgers move around during the day whilst in their sett. In Fig. 11 a), it can be seen that the two badgers are present in different parts of the sett. Fig. 11 (b) shows that badger B moves towards badger $\mathrm{C}$. Note that we know that this transition took place underground, as no radio beacons were detected by any of the surface nodes. In Fig. 11.c), it can be seen that the two badgers become co-located. This information can be used by zoologists to infer badger behaviour and interactions. Location information with this fine temporal resolution has never before been collected for badgers whilst underground. As such these findings help to elucidate research questions about underground badger behaviour.

\subsection{Tunnel visualization}

Once the locations of the badgers over time were determined, we used a simple method to reveal the structure of their tunnels. The locations were quantized into a grid with $30 \mathrm{~cm} \times 30 \mathrm{~cm}$ cells. We counted the number of times a badger was present in a cell to form a density map. Overall, 43 days of badger locations were collected. The aggregated locations of the badgers over time are shown in Fig. 12, using three different colourmaps. Dark regions indicate where a badger spent the majority of its time, whereas white regions represent unvisited areas. Using a linear colourmap, as in Fig. 12 a), very little of the sett structure is revealed, but locations where a badger was found to frequent are emphasized. Fig. 12 (b) shows the same data but plotted using a logarithmic colourmap. This captures all the locations visited, but does not show the locations most frequently visited with clarity. In Fig. 12 (c), a two tone binary colourmap is used, where any location visited is shaded in grey and any location where a badger was present more than $5 \%$ of the time is shaded in black. Thus, the same data can be used in different ways to understand badger habits and elucidate tunnel structure.

Notable is the narrow passage that can be seen to link the two bottom holes with the main chamber in Fig. 12 (c). This 


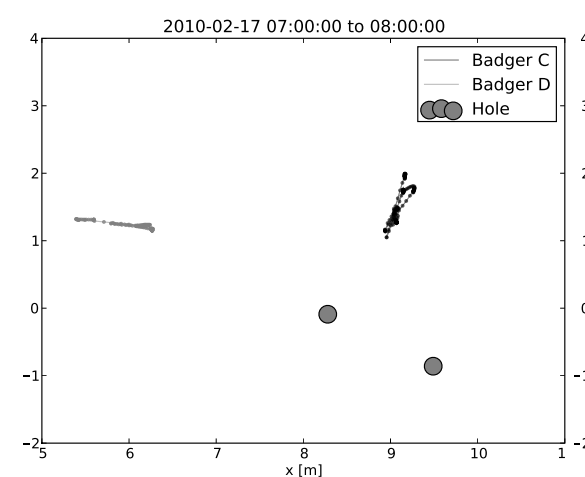

(a)

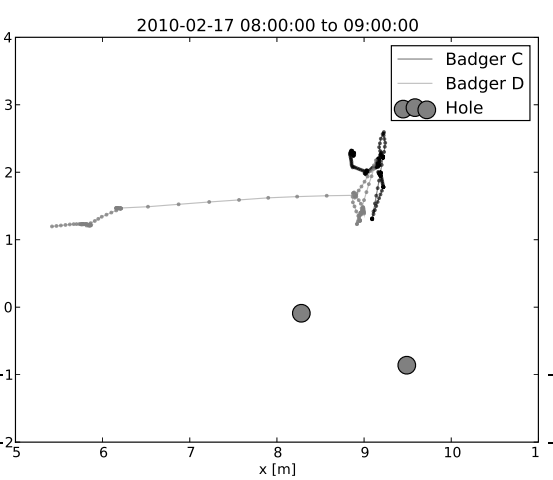

(b)

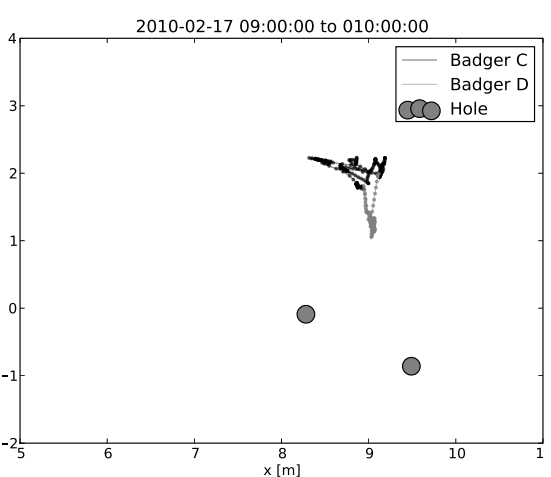

(c)

Figure 11. Hourly traces showing badger activity. (a) The two badgers are present in different chambers. (b) Badger D moves towards Badger $C$. (c) Badger $C$ and $D$ are now resident in the same location.

is significant, because it verifies that our position estimates link up with the holes that were surveyed manually. The tunnel visualizations show that there are two disjoint and unconnected chambers. We further validated this by observing that badgers never crossed between these areas without first going above ground (and being detected by the basestation because of beacon transmissions). Thus, although these holes were relatively closely spaced on the surface, they appear to lead to separate nests.

\subsection{Results from the surface sensor network}

In order to determine surface space use of the tagged badgers, 10 Tmote Sky nodes were placed approximately in a ring around the badger sett. In this application, we used proximity based detection as there was little overlap in the coverage between nodes, but it would be a simple matter to use one of the many existing RSSI based localization algorithms to improve the position accuracy in a denser deployment. A typical plot obtained from the surface tracking data is shown in Fig. 13 showing the movements of Badger C over a three hour time period. Initially, the badger has just entered the detection region. It then moves down towards the sett and enters the underground tunnel structure.

In summary, using the same animal tracking tag, we can seamlessly track the position of the animal both above and below ground. The two detection modalities (RF and MI) complement each other and allow us to provide high resolution data about badger behaviour without interruption.

\section{Related Work}

Wireless underground sensor/communication networks were proposed in 2006 [7], where the principle of using magnetic induction as an alternative to electromagnetic (EM) propagation was discussed, motivated primarily by the advantages of negligible variation in path loss due to water. The propagation of EM waves through soil with varying volumetric water content was experimentally measured in [15], where it was shown that water had a significant effect on the level of attenuation. An experimental validation of using low frequency UHF (433 MHz) for communication in underground-underground applications has been recently provided in [26]. This demonstrated that high power $(1 \mathrm{~W})$ commodity radio modules could be used to transmit information through $1 \mathrm{~m}$ of soil. However, to the best of our knowledge, there has yet to be an experimental demonstration of MI based communication in a sensor network context. The concept of using passive antenna coils as a magnetic waveguide in order to increase transmission range was been recently proposed [8]. This is an interesting approach as it shows how magnetic fields can be concentrated and guided without using additional power. We have demonstrated that not only is through-earth MI based communication feasible in a low power context, but also that it is possible to use the same technology for localization.

Magneto-inductive communication has been demonstrated in littoral (shallow water) environments, where traditional acoustic communication suffers heavily from fading [27]. Using a high transmitter power (200W), they were able to successfully communicate over a range of $300 \mathrm{~m}$. Low frequency through-the-earth (TTE) communication has also used by speleological groups for voice communication and rescue in caves [3].

The concept of MI localization itself is not new. Raab et al. in their seminal work present a magnetic localization system for determining the location and orientation of an object in 3-D space (i.e. six degrees of freedom) [22]. Commercial available products provide impressive accuracies (e.g. $10 \mathrm{~mm}, 1.5^{\circ}$ with a $100 \mathrm{~Hz}$ update rate) [4, 5]. However, the focus of these systems is on human-computerinterfacing, which requires high precision over a relatively small volume, typically within a $3 \mathrm{~m}$ radius. As such, power consumption is not a primary consideration and existing battery powered devices only last for a few hours. Our work has concentrated on reducing the power consumption to such an extent that long term operation on wild animals is a reality. Another approach which has been used in the medical field is tracking a magnetic "marker" [6]. The advantage of this is that the tag would be passive, however, individual animals could not be identified, limiting zoological applications. Additionally, operation has only been demonstrated over a very small volume $(60 \mathrm{~cm}$ x $60 \mathrm{~cm}$ x $30 \mathrm{~cm})$.

ZebraNet laid the groundwork for delay tolerant networking of animals [13]. This has been followed up by other sen- 


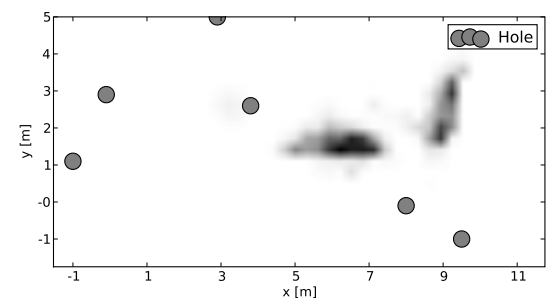

(a)

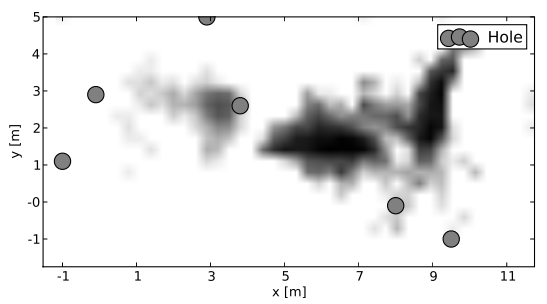

(b)

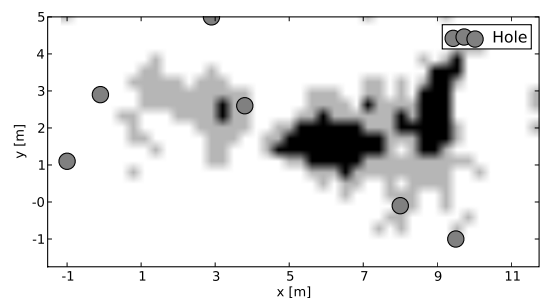

(c)

Figure 12. Density plot showing aggregated locations of badgers. (a) linear colourmap (b) logarithmic colourmap (c) two-tone threshold

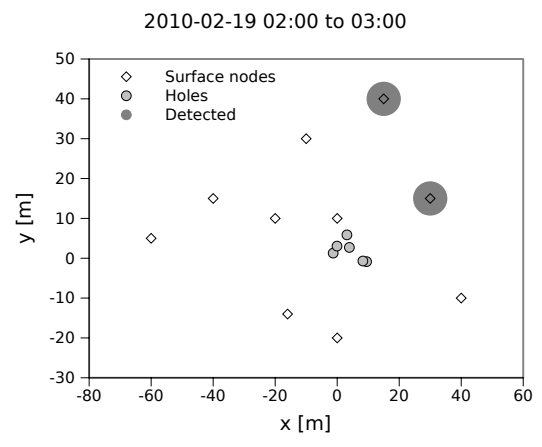

(a)

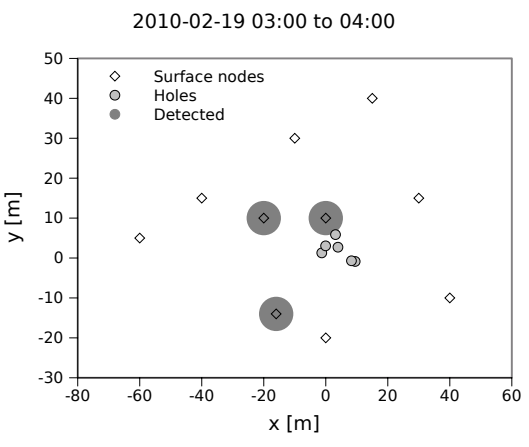

(b)

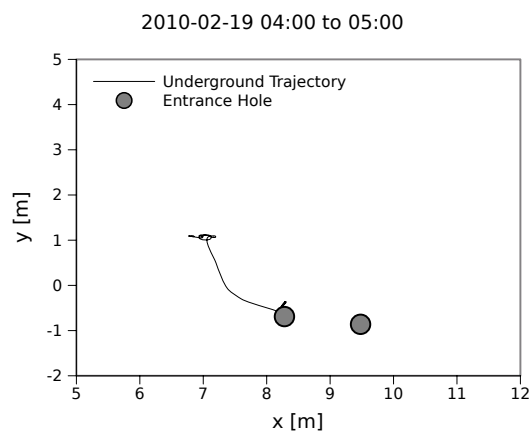

(c)

Figure 13. Example plot of badger activity over time, as captured by the surface tracking nodes. Nodes shaded gray have detected the badger. The centre of the sett is at the origin. (a) shows the badger entering the region. (b) shows the badger moving close to the sett. (c) shows the underground trajectory of the badger entering the sett.

sor network systems for tracking animals, such as TurtleNet [28]. Similar to our project, RatMote is a recent approach to the problem of determining the location of an underground animal, but the authors take an alternative approach to localization, using dead-reckoning [16]. The well known drawback of inertial tracking is that it is subject to drift, but they propose using SLAM to overcome these issues. However they have not yet presented any outdoor tracking results, and only laboratory trials. An additional disadvantage of inertial tracking is that it generates a vast amount of data in comparison to our method, which thus requires significant processing and data forwarding. MI also uses significantly less power than inertial tracking based techniques.

\section{Limitations and lessons learnt}

One of the main limitations of the present system is the limited range of detection, currently less than $10 \mathrm{~m}$. This is in part due to the small size of the detection coils that we use on the badger tag, in order to keep collar size and weight low. However, it is possible to detect the time varying magnetic field at a much greater distance by using a pickup coil with a larger area, e.g. by wrapping a coil around the badger's collar itself. To increase the range of the system, it would also be possible to use an additional gain stage to boost the signals detected by the transponders and use a matched filter approach, albeit at the cost of increased power consumption. Another option would be to increase the transmitter power. The fundamental issue remains however that the magnetic field falls off with an inverse cube law and thus is best suited to applications like underground tracking, where other approaches cannot be adequately used. There are also many contributing factors to localization error, including antenna geometry, coil shape and size, resonant frequency mismatch and background noise. These and other factors will be investigated further in future work.

A further approach to addressing the range limitations is to augment the tracking tag with an inertial sensing unit. Although this would increase collar cost and power consumption, this system could use magnetic fields to provide accurate localization in a small region and use inertial tracking to cover a larger area.

In the existing system we did not filter the position estimates. Using an approach such as an unscented Kalman Filter, which takes into account the non-linearity of the localization problem, positioning errors could be greatly reduced [14]. Again, fusing data obtained from other sensors, such as accelerometers, could even further enhance the accuracy of this technique.

Deploying devices on animals is not an easy task, as once they are attached, there is no possibility of retrieval. It is thus important to transmit diagnostic information so that problems can be resolved remotely. In this deployment, there was no possibility of reprogramming devices in the field. This could easily be incorporated, either by downloading data over traditional RF when above ground, or using MI communication whilst underground. This would have allowed us to modify code to perform tasks such as battery voltage 
logging (as opposed to just transmission).

\section{Conclusions}

This system demonstrates the first underground tracking of animals in their natural habitat, using custom built badger tags and magneto-inductive based localization. The method is non-invasive, autonomous and as a byproduct of localization also reveals the animal's tunnel structure. Although the tags did not meet their design lifetime of three months, they still have provided a wealth of data about badger behaviour underground at a resolution that has not been achieved previously. We also demonstrated how the technology can be augmented with information collected by a conventional, aboveground, sensor network. In summary, MI is a promising technique for ultra-low power localization of mobile sensor devices, with particular application to challenging environments.

\section{Acknowledgments}

This work was supported by EPSRC WildSensing grant (EP/E013678/1). Many thanks to Chris Newman, Christina Buesching and the members of the Wytham Badger Project core funded by the Peoples Trust for Endangered Species.

\section{References}

[1] http://www.sirf.com/,

[2] http://www.austriamicrosystems.com

[3] http://creg.org.uk/

[4] http://www.polhemus.com.

[5] http://www.ascension-tech.com/

[6] http://www.stl-gmbh.de/

[7] I. F. Akyildiz and E. P. Stuntebeck. Wireless underground sensor networks: Research challenges. Ad Hoc Networks, 4(6), 2006.

[8] I. F. Akyildiz, Z. Sun, and M. C. Vuran. Signal propagation techniques for wireless underground communication networks. Physical Communication, 2(3), 2009.

[9] J. Butler, T. Roper, and A. Clark. Investigation of badger (Meles meles) setts using soil resistivity measurements. Journal of Zoology, 232(3), 1994.

[10] A. Calderbank, I. Daubechies, W. Sweldens, and B. Yeo. Wavelet transforms that map integers to integers. Applied and Computational Harmonic Analysis, 5(3), 1998.

[11] G. Elkaim, E. Decker, G. Oliver, and B. Wright. Marine mammal marker (MAMMARK) dead reckoning sensor for in-situ environmental monitoring. Proc. of the ION/IEEE PLANS, 2006.

[12] R. Feynman. The Feynman lectures on physics. Addison Wesley Longman, 1970.

[13] P. Juang, H. Oki, Y. Wang, M. Martonosi, L. Peh, and D. Rubenstein. Energy-efficient computing for wildlife tracking: Design tradeoffs and early experiences with zebranet. ACM SIGOPS Operating Systems Review, 36(5), 2002.

[14] S. Julier and J. Uhlmann. A new extension of the Kalman filter to nonlinear systems. In Int. Symp. Aerospace/Defense Sensing, Simul. and Controls, volume 3, 1997.

[15] L. Li, M. C. Vuran, and I. F. Akyildiz. Characteristics of underground channel for wireless underground sen- sor networks. In Sixth Annual Mediterranean Ad Hoc Networking Workshop, Corfu, Greece, 2007.

[16] J. Link, G. Fabritius, M. Alizai, and K. Wehrle. BurrowView-Seeing the world through the eyes of rats. In Proc. of IEEE IQ2S, 2010.

[17] D. W. Macdonald and C. Newman. Population dynamics of badgers (Meles meles) in Oxfordshire, UK: numbers, density and cohort life histories, and a possible role of climate change in population growth. Journal of Zoology, 256, 2002.

[18] D. W. Macdonald, P. Riordan, and F. Mathews. Biological hurdles to the control of TB in cattle: A test of two hypotheses concerning wildlife to explain the failure of control. Biol. Conserv., 131(268), 2006.

[19] M. Misakian. Equations for the magnetic field produced by one or more rectangular loops of wire in the same plane. Journal Of Research-National Institute Of Standards And Technology, 105(4), 2000.

[20] W. E. Patitz, B. C. Brock, and E. G. Powell. Measurement of dielectric and magnetic properties of soil. Technical report, Sandia National Laboratories, New Mexico, 1995.

[21] N. Patwari, J. Ash, S. Kyperountas, A. Hero, R. Moses, and N. Correal. Locating the nodes: cooperative localization in wireless sensor networks. IEEE Signal processing magazine, 22(4), 2005.

[22] F. Raab, E. Blood, T. Steiner, and H. Jones. Magnetic position and orientation tracking system. Aerospace and Electronic Systems, IEEE Transactions on, AES15(5), Sept. 1979.

[23] T. J. Roper. Badger Meles meles setts - architecture, internal environment and function. Mammal Review, 22(1), 1992.

[24] L. M. Rosalino, D. W. Macdonald, and M. Santos-Reis. Activity rhythms, movements and patterns of sett use by badgers, Meles meles, in a mediterranean woodland. Mammalia, 69(3-4), 2005.

[25] A. Savvides, C. Han, and M. Srivastava. Dynamic fine-grained localization in ad-hoc wireless sensor networks. 2001.

[26] A. Silva and M. Vuran. Empirical Evaluation of Wireless Underground-to-Underground Communication in Wireless Underground Sensor Networks. In Proceedings of the 5th IEEE International Conference on Distributed Computing in Sensor Systems, 2009.

[27] J. J. Sojdehei, P. N. Wrathall, and D. F. Dinn. Magnetoinductive (MI) communications. In Oceans 2001, MTS/IEEE Conference, Honolulu, USA, 2001.

[28] J. Sorber, A. Kostadinov, M. Garber, M. Brennan, M. Corner, and E. Berger. Eon: a language and runtime system for perpetual systems. In Proceedings of the 5th international conference on Embedded networked sensor systems. ACM, 2007.

[29] E. Stuntebeck, D. Pompili, and T. Melodia. Underground wireless sensor networks using commodity terrestrial motes. Technical report, Georgia Institute of Technology, 2006. 